\title{
A Soft Drone with Multi-modal Mobility for the Exploration of Confined Spaces
}

\section{Conference Paper}

\section{Author(s):}

Fabris, Amedeo; Kirchgeorg, Steffen (D); Mintchev, Stefano (iD)

Publication date:

2021

Permanent link:

https://doi.org/10.3929/ethz-b-000513113

Rights / license:

In Copyright - Non-Commercial Use Permitted

Originally published in:

https://doi.org/10.1109/SSRR53300.2021.9597683

Funding acknowledgement:

ETH-15 20-2 - SquAshy - Soft Aerial robots that squeeze and crawl to traverse confined spaces (ETHZ) 


\title{
A Soft Drone with Multi-modal Mobility for the Exploration of Confined Spaces
}

\author{
Amedeo Fabris, Steffen Kirchgeorg, and Stefano Mintchev, Member, IEEE
}

\begin{abstract}
In post-disaster scenarios, rescuers are often confronted with the challenge of accessing confined and cluttered environments including long and narrow passageways, gaps in walls or ceilings. Because of their mobility and versatility, there is a growing interest in developing drones for the remote exploration of these dangerous and often difficult to access places. However, the mechanical design and locomotion strategies of current drones limit the size of the confined space that can be explored. In this work, we present a quadcopter capable of traversing long passageways $34 \%$ smaller than its nominal size. The combination of a soft morphing frame and multi-modal mobility allows the drone to exploit a new dynamic strategy for passageway traversal. The drone flies at a given speed towards the entrance of the passageway until it collides with it. The momentum and ability of the frame to soften allow the drone to passively fold and enter. Once the drone is squeezed between the walls of the passageway, it uses two tracks to crawl through. Through experiments, we characterize the main mechanical systems of the drone and study the entry into crevices of different sizes.
\end{abstract}

\section{INTRODUCTION}

In recent years, the exploration of confined artificial environments has become a major challenge. In search and rescue scenarios, the access of confined spaces such as narrow and long crevices, passageways, or gaps in walls and ceilings may be the only viable path to enter collapsed buildings and locate trapped people [1]. The remoteness, inaccessibility, and danger of these places have offered new challenges and applications for multirotors, which stand out in the field of robotics for their versatility and their ease of deployment [2].

To access confined environments, drones must traverse gaps and passageways that are often smaller than their nominal size. Recent works have proposed several methods for traversing such confined spaces by combining morphing capabilities with different perception, control, and planning strategies [3], [4], [5], [6], [7]. However, aerial locomotion sets a limit to the minimum size of the gap that a drone can traverse. Propellers need sufficient free space to rotate and the aerodynamic turbulence that they generate in proximity to surfaces hampers the flight in confined environments. Moreover, most of these morphing drones exploit a 'no touch' paradigm and are not equipped with adequate mechanical solutions to handle collisions, which are inevitable during locomotion in confined environments.

Animals have evolved more efficient and robust strategies for negotiating confined environments. These strategies

All the authors are with the Environmental Robotics Laboratory, Dept. of Environmental Systems Science, ETH Zurich, 8092 Zurich, Switzerland (e-mail: amedeo.fabris@ethz.ch, skirchgeorg@ethz.ch, smintchev@ethz.ch). The research was supported by the Swiss Center for Drones and Robotics of the Department of Defence, Civil Protection and Sport via armasuisse S+T under project n. 050-42, by the ETH Zurich Research Grants, and by the SNSF Eccellenza Grant PCEFP2/_186865.

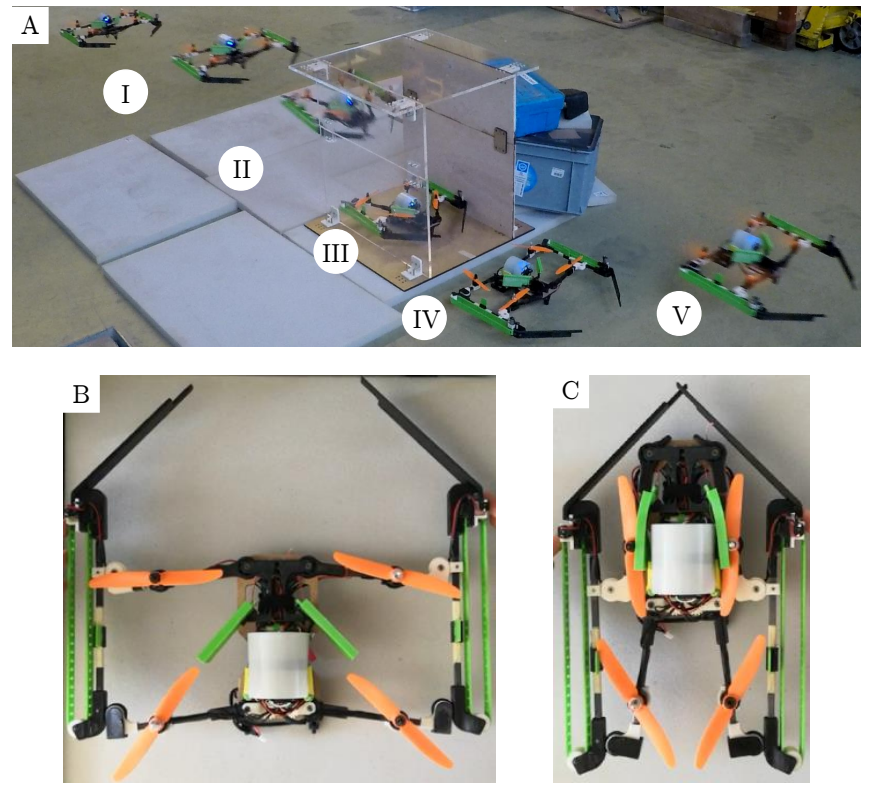

Fig. 1. Traversing narrow passageways with drones. The traversal is shown in the video of the supplementary material. (A) The proposed drone can halve the size of its soft body and use tracks to crawl through the passage. (B) Flight configuration and (C) folded configuration of the drone.

often combine multi-modal mobility with the softness of the animal's body for size reduction. Flying animals such as birds and insects actively or passively fold their wings when flying near or between obstacles [8], [9] and, if the environment is too cluttered, they also use their legs for locomotion. For instance, in the case of narrow and long passageways, goshawks not only fold their wings but also use their legs to facilitate the traversal and to push themselves out of the passageway [10]. In addition to multi-modal mobility, body morphology also plays an important role in the traversal of confined spaces. Cockroaches, for example, leverage their inherent soft bodies to flatten and traverse narrow crevices [11]. Moreover, the streamlined shape of their bodies improves traversability in densely cluttered terrains by facilitating penetration into gaps between vegetation [12].

Inspired by nature, we developed a drone where (i) adaptive morphology, (ii) soft behavior, and (iii) multi-modal mobility coexist. These features work in synergy to deliver a new dynamic strategy for the task of long and narrow passageway traversal with drones. In this approach, the drone flies at sustained speed against the confined space (Fig. 1A, I). The controlled collision of the drone with the entrance causes the frame to soften (Fig. 1A, II), and consequently to passively fold and squeeze inside the confined space (Fig. 1A, III). Once inside, the drone changes mode of locomotion and uses two lateral tracks to navigate through 
Proposed solution

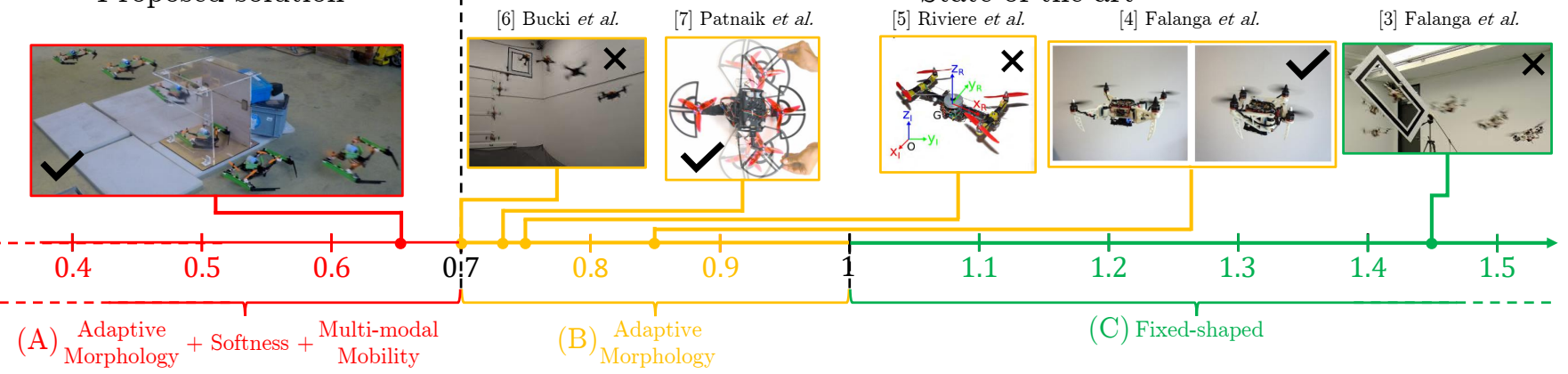

Fig. 2. Comparison of state-of-the-art methods for narrow gap traversal. The axis shows different values of $\beta$, a symbol in the image indicates the ability $(\checkmark)$ or inability $(\times)$ to traverse arbitrarily long passageways: (A) drones accessing confined spaces smaller than their nominal size leveraging multiple features simultaneously $\beta<0.7$; (B) drones relying on adaptive morphology only to access gaps smaller than their nominal size $0.7 \leq \beta<1 ;(\mathrm{C})$ fixed-shape drones only able to access gaps bigger than their nominal size $\beta>1$.

the passageway (Fig. 1A, III). On exit, the drone passively recovers its shape and can take off again (Fig. 1A, IV and V). We validate the effectiveness of our approach on a small scale manually flown quadrotor. The proposed strategy proves its effectiveness to access and navigate confined spaces down to $66 \%$ of the nominal width of the drone (Fig. 1B and 1C) without any rupture of the frame during the experiments.

In this work, Sec. II compares different state-of-the-art methods for gap traversal. Then, Sec. III, describes the proposed quadrotor and the adopted strategy for accessing confined spaces. In Sec. V, the preliminary experiments and the deployment of the drone are presented. Finally, in Sec. VI, we illustrate the conclusions and the outlook for future works.

\section{RELATED WORK}

To compare state-of-the-art methods for gap traversal (Fig. 2 ), we introduce what we call the gap-to-drone ratio $\beta$ :

$$
\beta=\frac{w_{g}}{w_{n}}
$$

where $w_{g}$ is the width of the gap that the drone aims to overcome, whereas $w_{n}$ is the nominal width of the drone (tip to tip) during flight. Therefore $\beta<1$ when the traversal strategy helps the drone in analysis to overtake a gap smaller than its nominal size (Fig. 2A and B). Whereas $\beta \geq 1$ implies that the traversal strategy exploited by the drone only allows the traversal of gaps bigger or equal to the size of the aerial vehicle in the nominal configuration (Fig. 2C).

Falanga et al. [3] propose a drone for traversing narrow gaps by leveraging the agile flight of a fixed-shape quadrotor. Although the high agility of the drone allows the traversal of inclined gaps, the drone can't reduce its size, which leads to $\beta>1$ (Fig. 2C).

To traverse gaps smaller than their size $(\beta<1)$, drones need an adaptive morphology to reduce their size. In [4] and [5], the authors rely on an active morphing mechanism to fold the arms of the drone (Fig. 2B). While the former drone [4] can overcome both horizontal and vertical gaps in a controlled fashion, i.e. by continuously maintaining stability during flight in all configurations, the latter [5] resort to a fully-aerial ballistic motion for traversing only vertical gaps. While both works achieve $\beta<1$, the fully-aerial ballistic traversal of Riviere et al. [5] offers better performance in terms of morphing capabilities with respect to [4] but at the expense of control loss on the roll axis during the maneuver. Since continuous stable flight is not achievable with the adopted change in morphology, the drone can reduce its size more, but the fully-aerial ballistic movement limits the length of the gap that can be traversed. In Bucki et al. [6], the drone uses a fully-aerial ballistic strategy as well for traversing vertical gaps, and achieves the best performance to date with a gap-to-drone ratio of $\beta=70 \%$ (Fig. 2B).

Since all these works avoid physical interaction with the environment, the size of the smallest traversable gap is increased by the addition of a safety factor on top of the width of the most compact configuration achievable by the drone during flight. This limitation is overcome by the work of Patnaik et al. [7], the authors present a quadrotor with passive adaptive morphology able to softly interact with the environment. During the traversal, the arms fold passively and the propeller guards rub against the surface of the passageway (Fig. 2B).

In all cases, the width and length of the traversable passageway remain limited by the use of aerial locomotion. The need to actively generate thrust during traversal requires space for the propellers to spin. In extremely folded configurations, propellers may overlap and reduce their thrust [13]. In this condition, the drone may struggle to sustain its weight. In addition, propellers suffer from ceiling effects and generate turbulence in proximity to surfaces that can disturb or even hamper the stable flight of the drone [14]. A fullyaerial ballistic traversal can overcome these limitations, but arbitrary long passageways cannot be traversed.

In this work, the current state-of-the-art in gap traversal will be challenged to access narrower (i.e. $\beta<0.70$ ) and arbitrarily long passageways. This result is achieved by adding the features of soft interaction and multi-modal mobility to the already established use of adaptive morphology (Fig. 2A).

\section{Traversal Methodology}

We propose a new dynamic strategy to traverse narrow and long passageways based on three main features (Fig. 3): (i) adaptive morphology of the drone frame, (ii) ability of the 

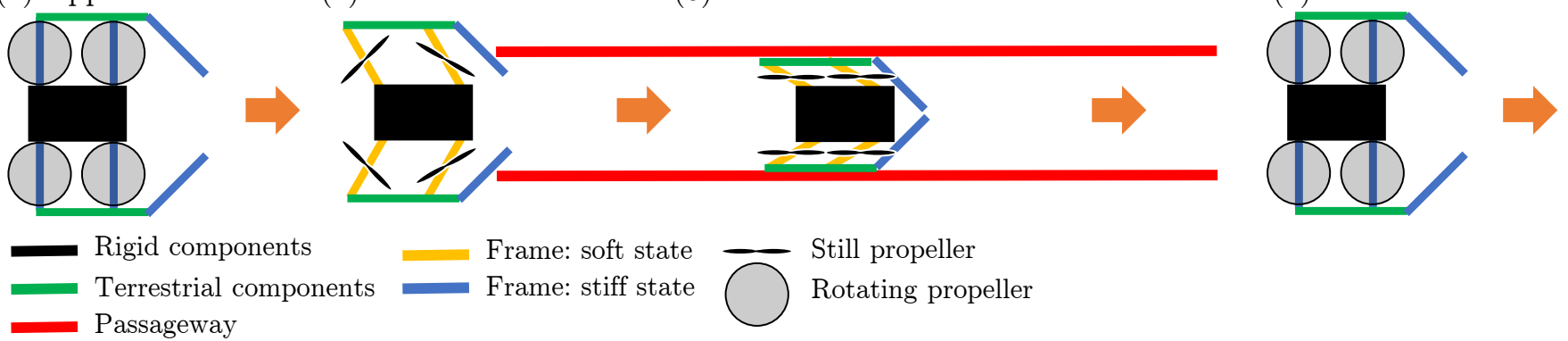

Fig. 3. Top view schematics of the dynamic strategy. The proposed approach leverages morphing capability, softness and multi-modal mobility simultaneously.

frame to soften, and (iii) multi-modal mobility. The strategy consists of four steps:

- Approach. The drone flies towards the center of the entrance of the passageway. The frame is in a rigid state to ensure stable and controlled flight (Step 1, Fig. 3).

- Entrance. The drone collides with the entrance of the passageway (Step 2, Fig. 3). The forces experienced by the drone during this controlled collision cause the frame to transition from a rigid to a soft state. The frame can now adapt its morphology and passively fold to reduce its size. The softening of the frame also reduces the risk of damages during the collision. The velocity of the drone before the impact confers a momentum that pushes the drone inside the passageway. The penetration is facilitated by the streamlined shape of the quadrotor, which also compensates for misalignment with the entrance of the passageway. Finally, the deceleration is detected by the onboard sensors and the system turns off the propellers and activates the tracks for terrestrial locomotion.

- Terrestrial Locomotion. After entering, the soft frame of the drone adapts to the size of the passageway and pushes the terrestrial appendages against the lateral walls. The drone can now crawl through the passageway (Step 3, Fig. 3).

- Exit. Once outside the passageway, the frame passively recovers the nominal morphology, switching back from the soft to the rigid state. At this point, the drone can take off and continue its mission (Step 4, Fig. 3).

Multi-modal mobility allows the drone to exploit the most appropriate locomotion strategy at every step. It can fly towards the entrance of passageways, and can then crawl inside where the aerial locomotion loses efficacy. The ability of the frame to soften is combined with its adaptive morphology. It allows the drone to withstand the controlled collision that is needed to squeeze and enter inside very narrow passageways. This passive morphing capability also benefits from lighter components as opposed to the ones needed in an active folding mechanism.

\section{DRONE DESIGN}

This section describes the mechanical design of the drone and provides an overview of the onboard electronics.

\section{A. Mechanical design}

The quadrotor consists of three main parts (Fig. 4): (i) a morphing frame with foldable arms; (ii) a central rigid body hosting the components allowing the frame to soften during the entry collision; (iii) a terrestrial locomotion system.

The adaptive morphology of the frame is implemented with two articulated parallelogram mechanisms composed of 3 hollow carbon rods each interconnected among them and to the central body thanks to $3 \mathrm{D}$ printed $\mathrm{ABS}$ revolute joints. A series of gears (Fig. 4A) synchronizes the folding of the two parallelogram mechanism (Steps 2 and 3, Fig. 3). Two frontal appendages trigger the folding of the frame during the impact of the drone with the entrance of the passageway. They consist of two 3D printed ABS flat structures with an inclination of $45^{\circ}$. During the folding of the frame, the spinning propellers are folded inwards with the potential to collide and damage parts of the drone as well as their blades (Figure 1A, II). To counteract this issue, two sets of flaps made out of a flexible filament (NinjaFlex, Fig. 4) are placed strategically to decelerate all propellers.

The central body of the drone is made out of smooth Medium-Density Fibreboard (MDF) and presents three holes to lighten the frame and facilitate cable management. While the rear part of the central body hosts all the electronics, the frontal part hosts the components that provide the dualstiffness behavior to the frame of the drone (Fig. 4B). The system consists of magnets and springs and takes inspiration from the solution proposed by the authors in [15], [16]. The magnets lock the parallelogram mechanism in the deployed configuration providing the rigidity necessary to fly in a stable and controlled manner. The magnets are strong enough to prevent the frame from folding due to the torque generated by the propellers, but they disengage when the drone collides with the entry to the passageway. The extension spring is connected to a pulley through an inextensible wire (SpiderWire, diameter $0.39 \mathrm{~mm}$ ) and resists the folding of the frame. The spring confers the soft behavior to the frame once the magnet is disengaged, partially absorbs the energy of the impact during the entrance maneuver, and presses the tracks against the side of the passageway. Finally, the two springs act as suspensions in case of obstacles or bumps encountered during the navigation inside the passageway and their exerted pressure is equally distributed thanks to the synchronized nature of the folding mechanism (Fig. 4A). It is important 


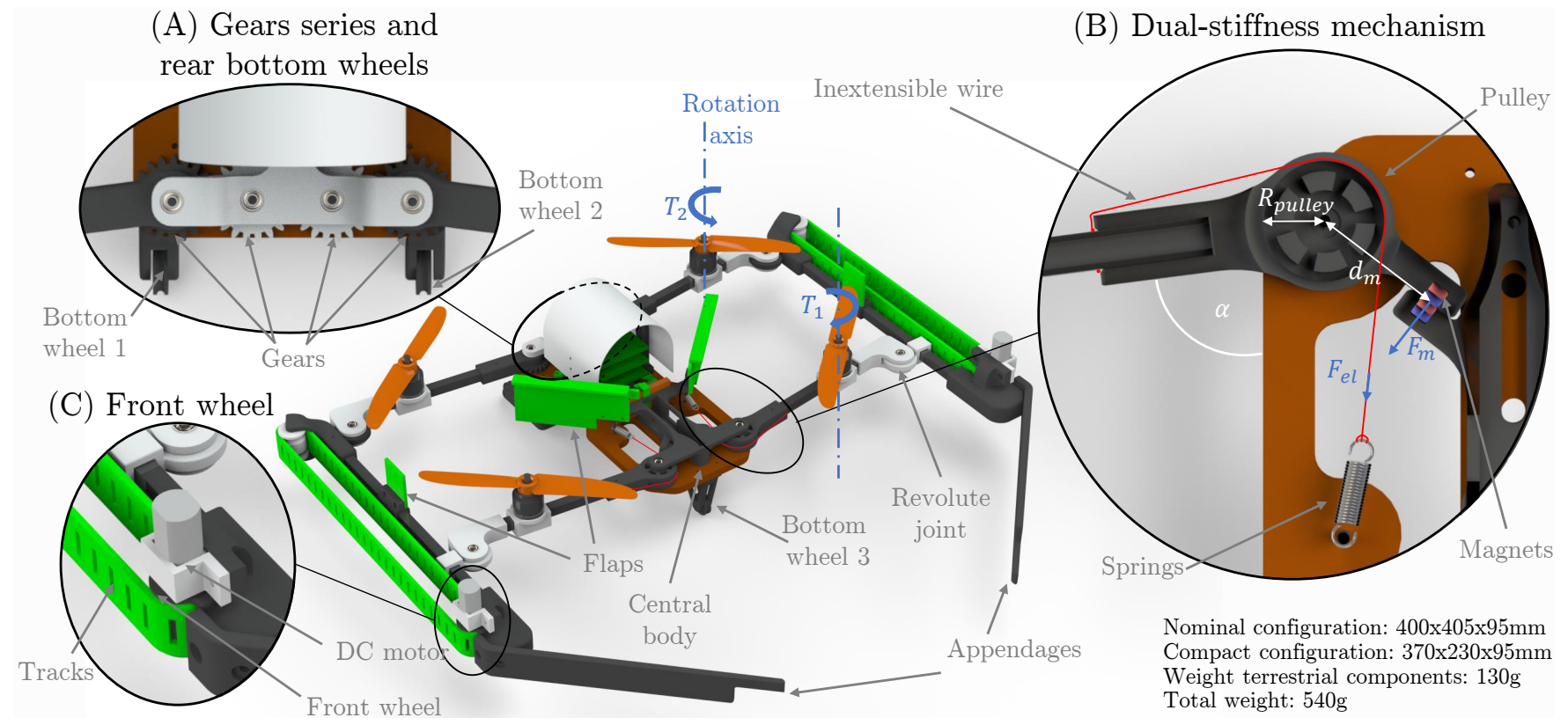

Fig. 4. Drawings and general specifications of the proposed drone. (A) Top view of the series of gears for the synchronization of the folding mechanism along with the rear bottom wheels; (B) top view of the section of the dual-stiffness mechanism; (B) zoomed-in view of the sprocket wheel for the terrestrial locomotion. At the bottom right corner of the image general characteristics of the drone are provided.

to note that, in the absence of this synchronization, the unbalanced pressure exerted on the sides of the passageway might hinder the drone to move forward and recover the nominal configuration during the exit maneuver.

The drone crawls inside the passageway with two driven tracks made out of NinjaFlex positioned on the sides of the drone. Their lateral location allows to leverage the inevitable contact with the wall of the passageway to move forward while guaranteeing a trade-off between the weight of active components and mobility inside the passageway. Their presence at the extremity of the frame can help pull the drone inside the passageway or push it out at the end of it. The tracks are driven from a pair of frontal sprocket wheels connected to a motor (Fig. 4C). To facilitate the locomotion on the bottom of the passageway, a set of three bottom wheels is installed beneath the main body of the drone, which also acts as a landing gear (Fig. 4).

\section{B. Electronics}

In flight mode, our quadrotor is driven by four 5030 twoblade propellers mounted on DYS BX1306 3100KV brushless DC motors. The motors are controlled by a Racerstar (35A BLheli_S 3-6S 4 in 1) Electronic Speed Controller (ESC), which receives the desired rotor speed commands from a mRo PixRacer R15 flight controller on which the PX4 firmware is flashed. The Pixracer board receives manual commands from a remote controller that is bound to a FrSky $\mathrm{XM}+\mathrm{RC}$ receiver. The autopilot is powered on by a Power Distribution Board (PDB) which is connected directly to the ESC. Both the ESC and the PDB are connected to a SWAYTRONIC LiPo battery 3S 11.1V 950mAh 35C / 70C. The stack of electronic boards is protected in the eventuality of collisions by a sheet of $0.4 \mathrm{~mm}$ thick fiberglass.

The terrestrial locomotion is made possible by a dual brushed ESC connected on one end to the PDB and the other end to two DC motors (250:1 Micro Metal Gearmotor HP $6 \mathrm{~V}$, Pololu, USA) that drive the tracks of the drone.

\section{RESULTS}

In this section, a description of the preliminary experiments is given along with the results of the deployment of the manually flown quadcopter.

\section{A. Dimensioning of the Dual-stiffness Components}

The magnets are selected to prevent the frame from folding during flight due to the torque applied by the propellers on the articulated parallelogram mechanism. With reference to Fig. 4, this condition can be computed considering the equilibrium equation of the mechanism:

$$
F_{m} d_{m} \geq T_{2}-T_{1}
$$

where $F_{m}$ is the holding force generated by the magnets, $T_{1}$ and $T_{2}$ are the torques of neighboring propellers on one side of the drone, and $d_{m}$ is the distance between the center of the pulley and the center of the magnet. In the worst-case scenario, when $T_{1}=0$ and $T_{2}$ assumes the maximum value of $0.03 \mathrm{Nm}$, the magnets need to generate an holding force of at least $F_{m}=130 \mathrm{~g}$ to prevent the folding of the frame. Therefore we selected magnets producing a force of $330 \mathrm{~g}$ which ensures a safety factor of 2.5 .

Three different springs were tested for the folding mechanism of the drone. As depicted in Fig. 5, each of these springs applies different torque profiles on the mechanism. Overall, the entry of the drone inside the passageway is facilitated by using weaker springs as they cause a low resistance to the folding of the drone. Weak springs require a lower speed to fold the frame of the drone and consequently low pitch angle which is desirable to facilitate the entry maneuver (see next section). However, they absorb less energy during 


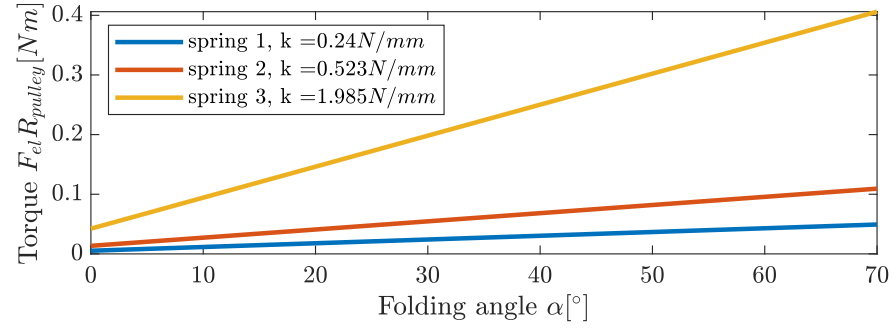

Fig. 5. Plot of the tested springs with their respective spring constants k. X-axis: folding angle $\alpha$ (Fig. 4B) of the drone; y-axis: elastic torque exerted by the spring with elastic force $F_{e l}$ and a pulley having radius $R_{\text {pulley }}=15 \mathrm{~mm}$.

the collision, thus leading to a faster folding of the arms forcing them to bounce on the main body of the aircraft. As a result, the arms quickly unfold. This motion causes the appendages to collide a second time against the sides of the passageway and the drone struggles to access the confined space. Oppositely, during the ground locomotion, stronger springs are more desirable. They help the tracks to exert more pressure against the walls of the passageway, thus increasing traction, as well as facilitate the drone's self-alignment with the passageway in case of a misaligned entrance. However, these springs require more energy to fold, therefore high velocities for successfully entering confined spaces which may lead to larger pitch angles hindering the entry inside the passageway. To find the right trade-off between entering feasibility and terrestrial locomotion resilience, we selected the tension spring 2 coupled with a pulley having radius $R_{\text {pulley }}=10 \mathrm{~mm}$.

\section{B. Experimental Characterization of the Entry Maneuver}

Before deploying the drone, we experimentally studied the maneuver to enter inside a horizontal passageway (Step 2, Fig. 3). This maneuver is very dynamic and its success is influenced by the speed of the drone $v$, its pitch angle $\theta$, the lateral misalignment with respect to the entrance $\Delta y$, and the width of the passageway $w_{g}$. We quantified the effect of these variables on the success of the maneuver through the experimental setup illustrated in Fig. 6 that allowed us to adjust each variable independently. The setup consists of a pendulum to launch the drone and a passageway with six varying widths and a fixed height. To select the horizontal speed of the drone upon impact with the passageway, we set the starting position of the pendulum at different inclinations $\gamma$ and we position the passageway next to the lowest point of the pendulum's motion. Moreover, a drone needs to pitch to move forward during flight. Therefore we equipped the pendulum with an adjustable end to launch the quadrotor with different pitch angles $\theta$. This variable is crucial as a significant change in pitch could lead the impact forces to not be entirely transmitted along the morphable direction of the drone, hampering its entry maneuver. The last parameter tested to study the entering maneuver is the maximum admissible lateral misalignments $\Delta y$ between the drone and the entrance of the passageway (Fig. 6, Top view). We launched the drone with different lateral misalignments ranging from $1 \mathrm{~cm}$ to $5 \mathrm{~cm}$. All these launches are performed for each of

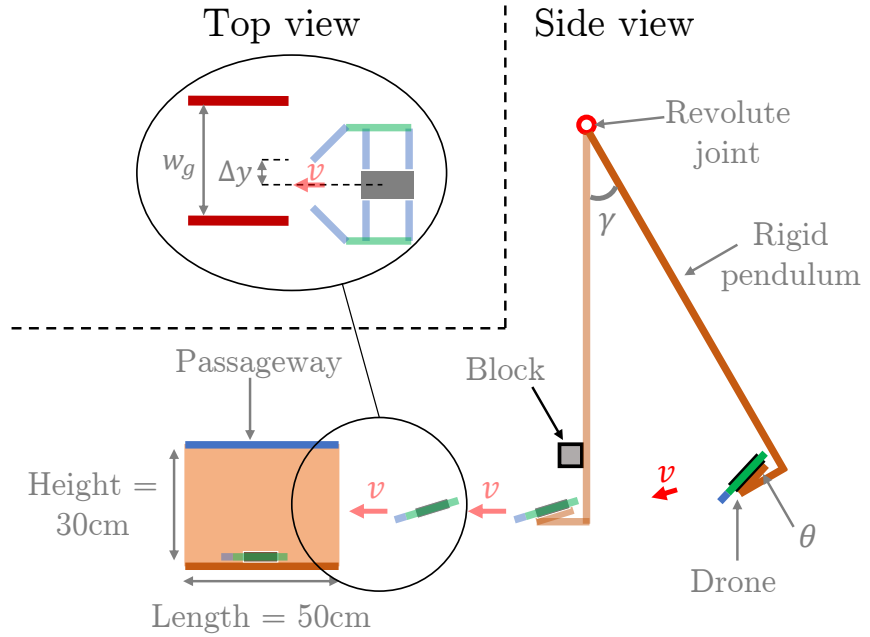

Fig. 6. Schematic view of the pendulum tests. The image also displays the definition of the variables of our experimental study. Some experiments of the entry maneuver are shown in the video of the supplementary material.

the six widths $\left(w_{g}\right)$ of the passageway. To have a statistical estimation of the successful entrance of the drone inside the passageway, each experiment is repeated three times. The entrance is considered to be successful if the quadrotor can engage with both tracks on the sides of the passageway in two launches out of three.

Fig. 7 summarizes the results of our experimental study of the drone in succeeding (green) or failing (red) to enter inside each of the six passageways. The maximum admissible misalignment $\Delta y$ in $\mathrm{cm}$ is specified for each successful entrance.

In general, the range of possible velocities and pitch angles to succeed in entering the passageways decreases from the largest passageway to the narrowest (Fig. 7, green vs red). Indeed, the narrowest passageway represents the folding limit of the platform. Furthermore, narrower passageways require higher speeds $(v>2 \mathrm{~m} / \mathrm{s})$ as the drone needs to fold more to enter the passageway successfully. On the other hand, a large pitch angle $\left(\theta=30^{\circ}\right)$ leads to failed entries. In this scenario, if the drone is too slow $(v<2 \mathrm{~m} / \mathrm{s})$ it struggles to fold its frame whereas if it is too fast it flips around the pitch axis. Although a lower pitch angle of $20^{\circ}$ already improves the entrance rate of the drone for all the passageways, the system still faces the issues discussed above but in a smaller range of velocities. On the contrary, for $\theta \leq 10^{\circ}$, we achieve very similar behavior in terms of successful entrance, enlarging the velocity envelope in which entering the passageway is possible.

Overall, by observing the tested lateral misalignment, the drone is more tolerant on larger passageways than it is on smaller ones, especially at low speeds (green values of $\Delta y$ in Fig. 7). Indeed, narrow passageways allow for smaller maximum misalignment. In this case, the wall of the passageway is more likely to enter and get caught between the appendages of the drone, rendering it unable to access the confined space. The same reasoning applies to bigger passageways, but with larger misalignment. Nevertheless, if the drone is launched against large passageways and $\Delta y \geq$ 


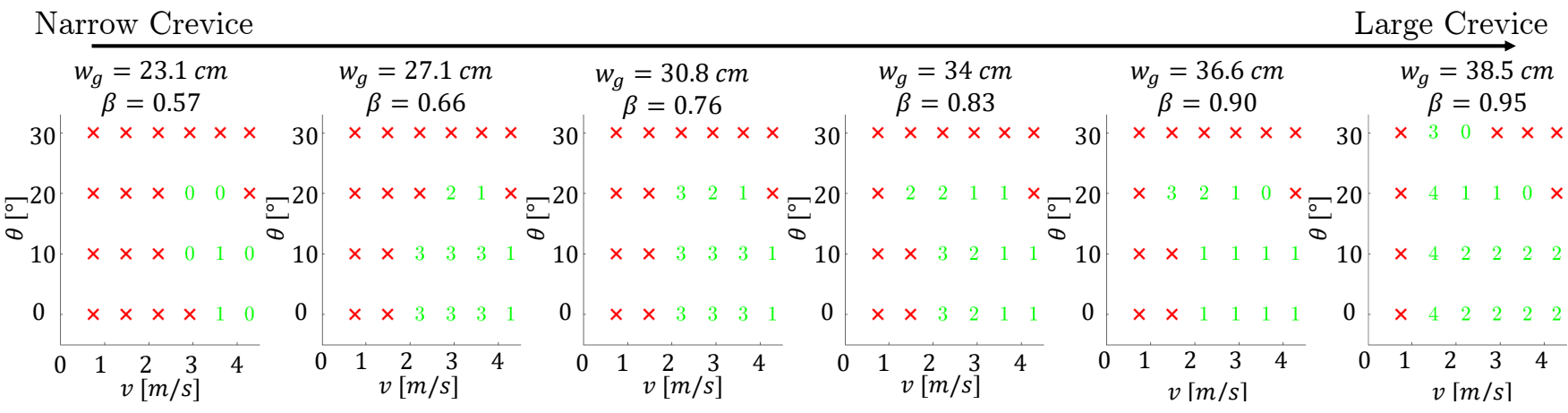

Fig. 7. Velocity envelope of the drone for a successful (green) and a failed (red) entering maneuver obtained with the selected spring. The maximum admissible misalignment $\Delta y$ in $\mathrm{cm}$ is specified for each successful entrance. The passageways are larger from left to right. $\mathrm{y}$-axis of the plots represent the pitch of the drone; $x$-axis of the plots represent the speed of the drone along the traversable direction of the passageway.

$5 \mathrm{~cm}$, it can hit a single wall of the passageway with only one of the appendages. In this scenario, if the speed of the drone is high $(v>2 \mathrm{~m} / \mathrm{s})$, the impact is enough to fold the drone. However, this single point of impact acts as a pivoting contact forcing the drone to rotate around the yaw axis. As a result, the quadcopter could land rotated with respect to the passageway unable to recover its alignment with respect to the passageway. For mild misalignments and speeds, this behavior may be counteracted by an asynchronous folding mechanism. In this scenario though, the system would struggle to move forward using its terrestrial locomotion due to the unbalanced outward pressure exerted on the side of the passageway.

Finally, if we consider only the launches executed with the selected spring and for low pitch angles (i.e. $\theta \leq 10^{\circ}$ ), the drone was able to enter successfully inside a given passageway with a success rate of $75 \%$ for all tested velocities.

\section{Manual Flight Tests}

To deploy our system, the same passageway as for the pendulum experiments was used. A chessboard is located in front of the passageway and allows to estimate the speed as well as the misalignments of the drone with respect to the passageway during the collision (Fig. 8, also shown in the video of the supplementary material).

Table I gives an overview of the results obtained during the deployment. All the passageways are listed from the narrowest to the largest with their respective width and gapto-drone ratio. The third column of the table highlights the success rate of the drone in entering the passageways during three manual flights. The second-last column shows the estimated speed of the drone along the traversable direction of the passageway averaged over all the collisions which led to a successful entrance. The last column shows the maximum misalignment that occurred with an entrance of the drone inside the confined spaces. Overall, the system had entered all the passageways except for the largest one and the narrowest one. Passageways having width $34 \mathrm{~cm}$, $30.8 \mathrm{~cm}$, and $27.1 \mathrm{~cm}$ were entered 2 times out of 3 , whereas the second largest passageway was accessed 1 time out of 3. The maneuver showed robustness to misalignment, and also to headings of the drone that were not perpendicular to the plane of the entrance of the passageway. Finally,

\begin{tabular}{ccccc}
\hline \multicolumn{5}{c}{ Deployment Overview } \\
\hline$w_{g}[\mathrm{~cm}]$ & $\beta$ & $\begin{array}{c}\text { Success } \\
\text { Rate }[\%]\end{array} v_{x_{\text {av }}}[\mathrm{m} / \mathrm{s}]$ & $\Delta y_{\max }$ \\
\hline 23.1 & 0.57 & 0 & $2.8 \pm 0.1$ & 3 \\
27.1 & 0.66 & 66 & $3 \pm 0.1$ & 3 \\
30.8 & 0.76 & 66 & $2.16 \pm 0.28$ & 4 \\
34 & 0.83 & 66 & $2.2 \pm 0.46$ & 3 \\
36.6 & 0.9 & 33 & $3 \pm 0.0$ & 0 \\
38.5 & 0.95 & 0 & $2.6 \pm 0.75$ & 6.5 \\
\hline
\end{tabular}

TABLE I

SUMMARY OF THE RESULTS OF THE DEPLOYMENT.

in all successful entries, the pitch at the moment of the collision was always less than $10^{\circ}$. Moreover, the drone showed mechanical resilience as it never broke during all the experiments except for some front propellers, but the integration of the soft flaps solved this issue.

Although the system showed resilience to small misalignments, the drone was not able to access the passageway with a misalignment $\Delta y \geq 6 \mathrm{~cm}$ as it was the case for all the flights against the widest passageway. In this scenario, the misalignment was also combined with a rolling maneuver at sustained speed (i.e. $v=2 \mathrm{~m} / \mathrm{s}$ ) causing the system to yaw during the collision. Nevertheless, these problems that affect the success rate of the proposed solution are mostly related to the inherent imprecision of the manual flight. As expected from the experiments in Sec. V-B, the entrance of the smallest passageway having $w_{g}=23.1 \mathrm{~cm}$ was very hard to achieve due to both the difficulty to center it in manual flight and also its very narrow dimension close to the smallest size of the drone. In this scenario, if the passageway was centered all the failed entries were caused by the arms bouncing on the main body of the drone which started to increase the width of the drone before entering inside the passageway. The confined space could be accessed with a higher speed of the drone $v>3 \mathrm{~m} / \mathrm{s}$ as highlighted by the pendulum tests. However, this target velocity is even more difficult to achieve in manual flight if combined with the requirement to center the entrance of the confined space.

Overall, the drone can access the passageways, also when slightly misaligned, with a pitch angle $\theta \leq 10$ and with a speed ranging from $1 \mathrm{~m} / \mathrm{s}$ up to $3 \mathrm{~m} / \mathrm{s}$ or more depending on the width of the confined space. To the best of the authors' knowledge, the system managed to improve the ability of the drone to access confined spaces by entering the passageway 


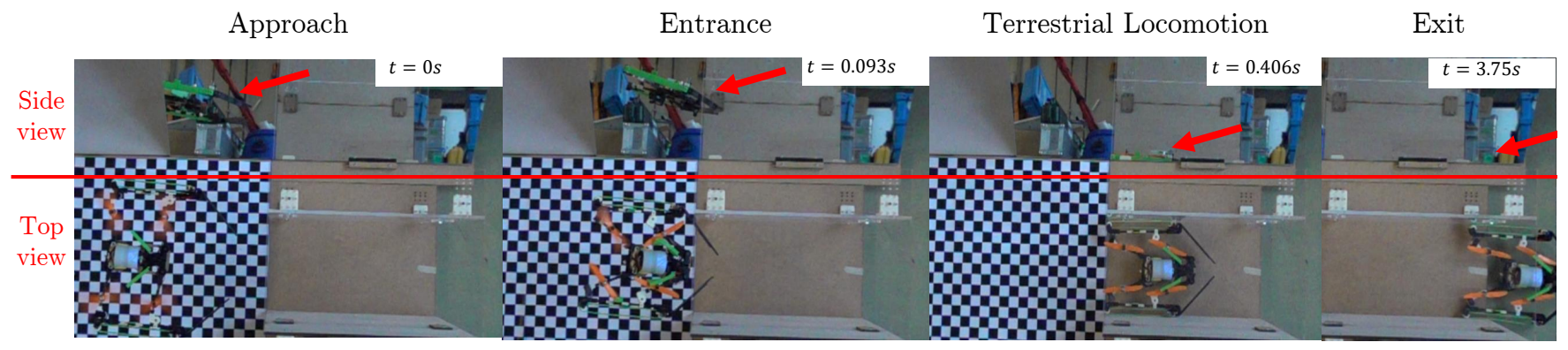

Fig. 8. Top view and side view sequence of a successful entrance in the passageway having $\beta=0.66$. The the experiment is filmed from above and the additional side view is possible thanks to an inclined mirror.

having $\beta=0.66$ during flight and $\beta=0.57$ during the pendulum tests.

\section{CONCLUSiOns AND Future Works}

In this work, we proposed a novel dynamic approach for drones to access and explore confined spaces. The combination of adaptive morphology, multi-modal mobility, and softness allows the drone to fly, squeeze, and crawl in narrow passageways. The versatility offered by the combination of these features is valuable not only for search and rescue, but also for other multi-domain tasks such as environmental monitoring [17] and infrastructure inspection [18]. The quadrotor achieve a state-of-the-art gap-to-drone ratio and performed multiple entries without permanent damages on the frame.

Although the current design proved to be a valuable proof of concept, the deployment of the drone highlighted some limitations. For instance, the drone can't exit from suspended passageways, i.e. passageways that aren't at ground level. In this scenario, the drone could be provided with the necessary propulsion to snap out of the passageway, unfold, and recover stable flight thanks to state-of-the-art control algorithms such as [19]. Moreover, significant misalignments of the drone with respect to the passageway as well as a wrong speed had often resulted in failed entries. In this regard, an optimal result could be obtained by equipping the quadrotor with the autonomy needed to center the passageway and remain within the velocity envelope of the experimental characterization of the entry maneuver. Finally, the terrestrial locomotion can be improved by adding active terrestrial components also beneath the drone making it more robust to misaligned entries.

\section{REFERENCES}

[1] J. Delmerico, S. Mintchev, A. Giusti, B. Gromov, K. Melo, T. Horvat, C. Cadena, M. Hutter, A. Ijspeert, D. Floreano, L. M. Gambardella, R. Siegwart, and D. Scaramuzza, "The current state and future outlook of rescue robotics," Journal of Field Robotics, vol. 36, no. 7, pp. 11711191, 2019.

[2] D. Floreano and R. J. Wood, "Science, technology and the future of small autonomous drones," Nature, vol. 521, no. 7553, pp. 460-466, 2015.

[3] D. Falanga, E. Mueggler, M. Faessler, and D. Scaramuzza, "Aggressive quadrotor flight through narrow gaps with onboard sensing and computing using active vision," Proceedings - IEEE International Conference on Robotics and Automation, pp. 5774-5781, 2017.

[4] D. Falanga, K. Kleber, S. Mintchev, D. Floreano, and D. Scaramuzza, "The Foldable Drone: A Morphing Quadrotor That Can Squeeze and Fly," IEEE Robotics and Automation Letters, vol. 4, no. 2, pp. 209216, 2019.
[5] V. Riviere, A. Manecy, and S. Viollet, "Agile Robotic Fliers: A Morphing-Based Approach," Soft Robotics, vol. 5, no. 5, pp. 541-553, 2018.

[6] N. Bucki and M. W. Mueller, "Design and control of a passively morphing quadcopter," Proceedings - IEEE International Conference on Robotics and Automation, vol. 2019-May, pp. 9116-9122, 2019.

[7] K. Patnaik, S. Mishra, S. M. R. Sorkhabadi, and W. Zhang, "Design and control of squeeze: A spring-augmented quadrotor for interactions with the environment to squeeze-and-fly," in 2020 IEEE/RSJ International Conference on Intelligent Robots and Systems (IROS). IEEE, 2020, pp. 1364-1370.

[8] C. D. Williams and A. A. Biewener, "Pigeons trade efficiency for stability in response to level of challenge during confined flight," Proceedings of the National Academy of Sciences, vol. 112, no. 11, pp. 3392-3396, 2015.

[9] A. M. Mountcastle and S. A. Combes, "Biomechanical strategies for mitigating collision damage in insect wings: structural design versus embedded elastic materials," Journal of Experimental Biology, vol. 217, no. 7, pp. 1108-1115, 2014.

[10] B. T. T. A. G. to Britain, "Goshawk flies through tiny spaces in slomo!" published on 05-05-2011. https://www.youtube.com/watch?v= 2CFckjfP-1E, visited on 28-09-2020.

[11] K. Jayaram and R. J. Full, "Cockroaches traverse crevices, crawl rapidly in confined spaces, and inspire a soft, legged robot," Proceedings of the National Academy of Sciences of the United States of America, vol. 113, no. 8, pp. E950-E957, 2016.

[12] C. Li, A. O. Pullin, D. W. Haldane, H. K. Lam, R. S. Fearing, and R. J. Full, "Terradynamically streamlined shapes in animals and robots enhance traversability through densely cluttered terrain," Bioinspiration and Biomimetics, vol. 10, no. 4, p. 46003, 2015.

[13] A. Fabris, K. Kleber, D. Falanga, and D. Scaramuzza, "Geometryaware compensation scheme for morphing drones," arXiv preprint arXiv:2003.03929, 2020.

[14] A. E. Jimenez-Cano, P. J. Sanchez-Cuevas, P. Grau, A. Ollero, and G. Heredia, "Contact-based bridge inspection multirotors: Design, modeling, and control considering the ceiling effect," IEEE Robotics and Automation Letters, vol. 4, no. 4, pp. 3561-3568, 2019.

[15] S. Mintchev, S. de Rivaz, and D. Floreano, "Insect-inspired mechanical resilience for multicopters," IEEE Robotics and automation letters, vol. 2, no. 3, pp. 1248-1255, 2017.

[16] S. Mintchev, J. Shintake, and D. Floreano, "Bioinspired dual-stiffness origami," Science Robotics, vol. 3, no. 20, 2018.

[17] A. Nakamura, R. L. Kitching, M. Cao, T. J. Creedy, T. M. Fayle, M. Freiberg, C. N. Hewitt, T. Itioka, L. P. Koh, K. Ma, Y. Malhi, A. Mitchell, V. Novotny, C. M. Ozanne, L. Song, H. Wang, and L. A. Ashton, "Forests and Their Canopies: Achievements and Horizons in Canopy Science," Trends in Ecology and Evolution, vol. 32, no. 6, pp. 438-451, 2017.

[18] H. Shakhatreh, A. H. Sawalmeh, A. Al-Fuqaha, Z. Dou, E. Almaita, I. Khalil, N. S. Othman, A. Khreishah, and M. Guizani, "Unmanned Aerial Vehicles (UAVs): A Survey on Civil Applications and Key Research Challenges," IEEE Access, vol. 7, pp. 48 572-48 634, 2019.

[19] M. Faessler, F. Fontana, C. Forster, and D. Scaramuzza, "Automatic reinitialization and failure recovery for aggressive flight with a monocular vision-based quadrotor," in 2015 IEEE international conference on robotics and automation (ICRA). IEEE, 2015, pp. 1722-1729. 